\title{
Low intensity Extra Corporeal Shock Wave Therapy in Patients with Erectile Dysfunction: Our experience in ACKU
}

\author{
Md. Fazal Naser1 ${ }^{1}$, Mahmood Hasan ${ }^{2}$, Md. Waliul Islam ${ }^{1}$, Tajkera Sultana Chowdhury ${ }^{3}$, Md. Shohrab Hossain ${ }^{4}$
}

Received: 02 - 03 - 2020

Accepted: 05 - 05 - 2020

Conflicts of interest: None

Keywords: Penis; Erectile dysfunction (ED); Low intensity extra corporeal shockwave therapy (Li-ESWT). good outcomes reported. subjects respectively.

\begin{abstract}
Introduction: Oral 5-phosphodiesterase inhibitors (PDE5-I) is the main therapeutical options in erectile dysfunction (ED). It shows good results, but non-responders lack other effective options and its effect is also not long lasting. Since last few years, low-intensity extracorporeal shockwave therapy (Li-ESWT) in the corpora cavernosa showing promising results. This article presents our early experiences in Advanced Center of Kidney disease and Urology (ACKU) with the aim to evaluate clinical efficacy of Li-ESWT.
\end{abstract}

Materials and methods: Thirty four patients with ED were prospectively included in the study during the period of January 2018 to Jun 2019.Treatment was performed using the PiezoWave2 (Richard Wolf, Germany) device with a linear probe. Treatment protocol included a weekly session for four weeks. Each session delivered 2000 shocks on the perineum plus 4000 shocks on dorsum penis with an energy flux density (EFD) of $0.160 \mathrm{~mJ} / \mathrm{mm}^{2}$. Every patient has been re-evaluated 1.5 and 3 months after the last session. Pre-and post-procedure International Index Erectile Function - Erectile function domain (IIEF-EF) score, Erection Hardness Score (EHS) and Global Assessment Questionnaire-Question 1 (GAQ-Q1) answers were obtained.

Results: Mean age of the study population was $39.4( \pm 12.9)$ years, $35.29 \%$ diabetic, $20.59 \%$ with hypertension and $55.85 \%$ smokers. Mean baseline IIEF-EF was 14.6, at 6 week post LiSWT was 16.4 ( $p>0.05)$ and at 3 months post LiSWT was $19.2(p<0.05)$. EHS was significantly improved at 3 month in comparison to baseline $(p<0.05) .20 .59 \%$ patients answered positively to GAQ-Q1 at 6 week and $61.76 \%$ at 3 months. IIEF-EF score change of $>5$ and increase of EHS $>2$ were observed in $62.88 \%$ and $70.59 \%$ study

Conclusions: $L i$-ESWT is a safe, harmless and repeatable treatment tool for ED with

1. Associate Professor of Urology, Shaheed Suhrawardy Medical College and Consultant Urologist, Advanced Centre of Kidney and Urology (ACKU), Shyamoli Dhaka, Bangladesh.

2. Assistant Registrar of Urology, Shaheed Suhrawardy Medical College Hospital and Consultant Urologist, Advanced Centre of Kidney and Urology (ACKU), Shyamoli Dhaka, Bangladesh.

3. Assistant Professor of Urology, Shaheed Suhrawardy Medical College and Consultant Urologist, Advanced Centre of Kidney and Urology (ACKU), Shyamoli Dhaka, Bangladesh.

4. Professor of Urology, Dhaka Central International Medical College and Consultant Urologist, Advanced Centre of Kidney and Urology (ACKU), Shyamoli Dhaka, Bangladesh.

Correspondence : Dr. Md. Fazal Naser, Associate Professor of Urology, Shaheed Suhrawardy Medical College and Consultant Urologist, Advanced Centre of Kidney and Urology (ACKU), Shyamoli Dhaka-1207, Bangladesh. Email : fazaltamim01@yahoo.com 


\section{Introduction:}

Erectile dysfunction (ED) is not an uncommon cause of sexual disorder in men occurring with 1 in 5 US male populations ${ }^{1}$. It is defined as the inability to achieve or maintain a penile erection satisfactory for sexual intercourse. Although vasculogenic ED is the most common type, there exist some other etiological types of ED like psychogenic, neurogenic, endocrine and drug-induced $\mathrm{ED}^{2}$. In spite of its high prevalence; the options for the treatment of ED are limited. With the introduction of Phosphodiesterase type-5 inhibitor (PDE5i) in the world market in 1998, a revolutionary change has been observed in this field because approximately $60 \%$ of patients could recover their erectile function and could lead a satisfactory sex life ${ }^{3}$. For men who do not respond to these oral agents, vacuum erection devices, urethral suppositories, intracavernosal injections, and penile prostheses can provide satisfactory alternatives. Intracorporeal injection of various vasoactive agents is popular but is not very patient friendly. Vacuum devices and penile prosthesis also have limitations. Even PDE5I have significant limitations; namely, planned intercourse and only $60 \%$ response rates ${ }^{1,2}$. Furthermore, PDE5I are associated with wide spectrum of side-effects.

In this backdrop, different trials have been conducted with the use of low-intensity shock wave treatment (SWT) in men with erectile dysfunction. But large scale data regarding its efficacy are scarce. Since the 1980s, shockwaves of different intensities have been used therapeutically in medicine. High-intensity shockwaves (pressure 1/4 450 bar) have been implemented in the treatment of urolithiasis, mediumintensity shockwaves (pressure $1 / 4200$ bar) in the treatment of arthralgia, tendinitis, and bursitis, and more recently LISWT (pressure $1 / 480$ bar) in the treatment of $\mathrm{ED}^{4}$. Young and Dyson discovered that therapeutic ultrasound encourages angiogenesis by enhancing the expression of vascular endothelial growth factor ${ }^{5}$. The idea of applying LISWT to the penis came out from a study with animals that proved that the energy of shockwaves applied to the myocardium of pig's improved ischemia-induced myocardial dysfunction ${ }^{6}$. By extrapolating these findings to ED, it was presumed that shockwaves applied to the penis might increase blood flow and improve endothelial function through the stimulation of angiogenesis in the corpus cavernosum. Despite these experiments in animal model, the exact mechanism of action is still not completely elucidated. However, low-intensity energy has been shown to induce the production of a physiologically significant amount of non-enzymatic nitric oxide and activate intracellular cascade pathways that trigger the release of angiogenic factors ${ }^{7}$. We do here describe our initial short term experience on treating ED patients with ESWT after being inspired by some successful projects performed in different parts of the world in this field $^{4,8,9,10}$.

\section{Materials and method:}

The study was conducted in Advanced Center of Kidney disease and Urology (ACKU) during the period of January 2018 to Jun 2019. Ethical permission was taken from the ethical committee of Dhaka Central International Medical College (DCIMC) ) as ACKU is a part of DCIMC. This was a single centered, single armed, uncontrolled, prospective type of observational study. 34 patients complaining of ED during consultation at our outpatient clinic were offered to participate in the study. During the first visit, subjects were screened according to the eligibility criteria and filled out the erectile function domain of the International Index of Erectile Function (IIEF-EF) questionnaire. We obtained the subjects' medical history and performed a physical examination. All subjects consented not to use other therapies for ED during the study period. Participants previously treated for ED ceased therapy 4 weeks before entering the study. Detailed inclusion and exclusion criteria are listed in Table 1.

Table 1: Inclusion and exclusion criteria.

\begin{tabular}{ll}
\hline Inclusion criteria & \multicolumn{2}{l}{ Exclusion criteria } \\
\hline 1. Age $>25 \mathrm{y}$. & 1. Surgery or radiotherapy of pelvic region. \\
2. Complaining of ED $>6 \mathrm{mo}$. & 2. Treatment with anticoagulants. \\
3. In stable relationship $(>3 \mathrm{mo})$. & 3. Treatment with anti androgens. \\
& 4. Anatomic penile deformation or penile prosthesis. \\
& 5. Total testosterone level $<8 \mathrm{nmol} / \mathrm{dl}$. \\
& 6. Serious heart or lung disease. \\
& 7. Psychiatric or neurologic disorder. \\
& 8. Pregnant partner. \\
& 9. IIEF-EF score $\geq 25$. \\
\hline
\end{tabular}


Each participant received weekly treatment sessions for 4 weeks. Each session lasted approximately 30 minutes and delivered 2000 shocks on the perineum plus 4000 shocks on dorsum penis with an energy flux density (EFD) of $0.160 \mathrm{~mJ} / \mathrm{mm} 2$ by using a piezoelectric linear therapy source (FBL10, RichardWolf $\mathrm{GmbH}$, Knitlingen, Germany). Every patient was re-evaluated at 6 week and 3 months after the last session. At baseline they completed the IIEF-EF and Erection Hardness Scale (EHS) questionnaires. Postprocedure IIEF-EF, EHS and Global Assessment Questionnaire-Question 1 (GAQ-Q1) answers were obtained. After the treatment each participant was also asked about any side-effects. Subjects completed the questionnaires with the help of a male research nurse in a separate room and were not disturbed by other participants or investigators. To enable comparison of our findings with results of other trials ${ }^{11,12,13}$ we defined our trial as successful when there is changes in IIEF-EF score of at least 5 points and increase of EHS score to at least 3 points.

\section{Results:}

Mean age of the study population was $39.4( \pm 12.9)$ years, $35.29 \%$ diabetic, $20.59 \%$ with hypertension and $55.85 \%$ smokers. Mean baseline IIEF-EF was 14.6, at 6 week post Li-ESWT was $16.4(\mathrm{p}>0.05)$ and at 3 months post LiSWT was $19.2(\mathrm{p}<0.05)$. EHS was significantly improved at 3 month in comparison to baseline $(\mathrm{p}<0.05) .20 .59 \%$ patients answered positively to GAQQ1 at 6 week and $61.76 \%$ at 3 months. IIEF-EF score change of $>5$ and increase of EHS $>2$ were observed in $62.88 \%$ and $70.59 \%$ study subjects respectively.

Table II : Demographic and clinical characteristics of the study subjects.

\begin{tabular}{lc}
\hline Age $(\mathrm{y})$, mean $( \pm \mathrm{SD})$ & $39.4( \pm 12.9)$ \\
$\mathrm{BMI}(\mathrm{kg} / \mathrm{m} 2)$, & $26.7( \pm 3.8)$ \\
Smoker, $\mathrm{n}(\%)$ & $19(55.85)$ \\
Hypertension, $\mathrm{n}(\%)$ & $07(20.59)$ \\
Diabetes, n $(\%)$ & $12(35.29)$ \\
Total testosterone (nmol/dL), mean $(\mathrm{SD})$ & $19.4( \pm 5.7)$ \\
ED duration (months), mean (range) & $57(12-108)$ \\
Effect of previous treatment with PDE-5i, $\mathrm{n}(\%)$ \\
$\quad$ Responders & $19(55.86)$ \\
$\quad$ Non-responders & $12(35.19)$ \\
$\quad$ Never used & $03(08.82)$ \\
\hline
\end{tabular}

$\mathrm{BMI}=$ body mass index; PDE-5i = phosphodiesterase type 5 inhibitor.

$\mathrm{SD}=$ Standard Deviation

Table 3 : International Index Erectile Function (IIEF-5) scores and Global Assessment Questionnaire-Question 1 (GAQ-Q1).

\begin{tabular}{lccccc}
\hline Variable & Baseline & At 6 week & At 3month & P value $^{*}$ & P value $^{\#}$ \\
\hline IIEF-ED scoremean $( \pm$ SD) & $14.6( \pm 8.80)$ & $16.2( \pm 4.70)$ & $19.4( \pm 7.20)$ & 0.416 & 0.016 \\
EHS scoremean $( \pm$ SD) & $1.21( \pm 0.52)$ & $2.1( \pm 0.81)$ & $2.42( \pm 0.80)$ & 0.072 & 0.0001 \\
\hline
\end{tabular}

* Difference between baseline and 6 week score

\# Difference between baseline and 3 month score

Table 4 : Result of Global Assessment QuestionQuestion 1 (GAQ-Q1*).

\begin{tabular}{lccr}
\hline GAQ-Q1 & At 6 week & At 3month & P value \\
\hline Positive response, & $07(20.59)$ & $21(61.76)$ & 0.0012
\end{tabular}

$\mathrm{n}(\%)$

Negative response, $27(79.41) \quad 13(38.24)$

$\mathrm{n}(\%)$

*GAQ-Q1: Has the treatment you have been taking improved your erectile function?

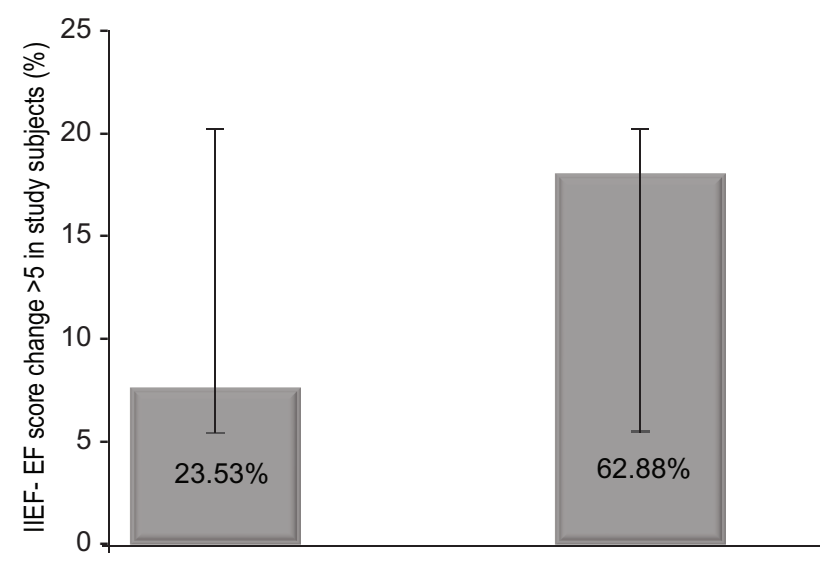

Fig.-1: IIEF-EF score change $>5$ in study subjects. 


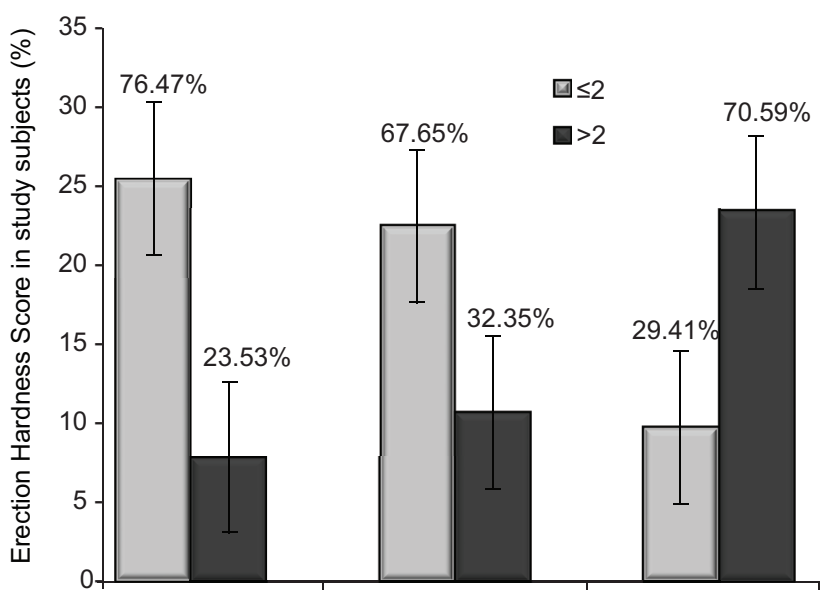

Fig.-2: Erection Hardness Score in study subjects.

\section{Discussion:}

This study represents the efficacy of linier focused LIESWT to treat ED. We were able to demonstrate a significant improvement in IIEF-IF and EHS at three month of post procedure follow up. Erectile function was recovered in about $60 \%$ of patients after treatment with LISWT. Most randomized, double-blinded, shamcontrol trials have reported the efficacy of LISWT in patients with ED and our results are almost in consistent with them ${ }^{11,13,14}$. Vardi et al demonstrated that LISWT had a positive short-term clinical and physiologic effect on the erectile function of men who respond to oral PDE5i therapy. They found a significantly greater increase in the IIEF-EF score in the treated group than in the sham-treated group ${ }^{14}$. However, Yee et al, did not find significant statistical evidence in the IIEF score and EHS score in a group of 28 patients under LISWT treatment compared with a sham-treated group of 30 patients. However, they found a significant difference in patients with a subgroup of patients-severe ED, according to the Sexual Health Inventory for Men and concluded that LISWT has clinical efficacy in this subgroup of patients ${ }^{11}$. More recently, Srini et al, in a randomized double-blinded trial with active treatment and sham therapy, reported a positive long term efficacy in patients with vasculogenic ED treated with linear focused shockwaves ${ }^{13}$. In a narrative review of all published studies, Gruenwald et al. found that $60 \%$ to $75 \%$ of treated patients who responded to PDE5i therapy could eliminate their dependency on those drugs and achieve an erection and vaginal penetration and that $72 \%$ of non-responders to PDE5i before undergoing LISWT became responders and achieved vaginal penetration ${ }^{10}$. It is well known that changes in IIEF imply only an improvement in score but does not necessarily guarantee a patient's successful or complete sexual intercourse. So, improvement of IIEF score cannot be the only determinant of evaluation of ED treatment. To overcome this limitation we used EHS and GAQ-Q1 to asses our patients. Sixty percent of the patients of the present study achieved and maintained an erection after penetration, and they were satisfied with the improvement of their penile rigidity after treatment.

This study has several limitations that are important to consider. First, its lack of a placebo group that prevents a proper comparison of the effects of LIWST. Second, this research extended through a follow-up period of only 03 months which may not be adequate to construct a meaningful conclusion. Third, there is no certainty that these improvements were due to the vascular changes suggested by other investigators because this study had an observational design of clinical practice; patients did not undergo any penile vascular study such as a Doppler evaluation during follow up that could show changes in the cavernosal arteries. Fourth, regarding the uncertainties to LISWT; it is not clear whether the number of sessions and treatments was sufficient. It does not define the best profile of patients who might benefit from this treatment. The mechanism of action is also not clear. However, LISWT has a good safety profile, with no adverse events reported.

In contrast, whenever independent pilot studies are conducted, the number of patients included tends to be small, and the results cannot be generalized. Although our data are limited here, the experiences reported in the literature thus far, one can consider these data quite promising. This new treatment modality seems promising to optimize treatments of ED.

\section{Conclusion:}

The present study showed the Li-ESWT was effective in a significant proportion of patients, and it was assumed as tolerable and safe with a relatively short follow up. Further validation with respect to such treatment's optimal targets and ideal protocol require more studies to arrive at a conclusion. In the future, this could be one of the few non-pharmacological ED treatment modalities. 


\section{References:}

1. Wessells H, Joyce GF, Wise M, Wilt TJ. Erectile dysfunction. J Urol, 2007; 177:1675-1681.

2. Shamloul R, Ghanem H. Erectile dysfunction. Lancet, 2013; 381:153-165.

3. Eardley I, Donatucci C, Corbin J, et al. Pharmacotherapy for erectile dysfunction. J Sex Med, 2010; 7:524-540.

4. Bechara A, Casabé A, De Bonis W, and Ciciclia PG. Twelve-Month Efficacy and Safety of LowIntensity Shockwave Therapy for Erectile Dysfunction in Patients. Who Do Not Respond to Phosphodiesterase Type 5 Inhibitors. Sex Med, 2016; 4:e225ee232.

5. Young SR, Dyson M. The effect of therapeutic ultrasound on angiogenesis. Ultrasound Med Biol, 1990; 16:261-269.

6. Nishida T, Shimokawa H, Oi $\mathrm{K}$, et al. Extracorporeal cardiac shock wave therapy markedly ameliorates ischemia-induced myocardial dysfunction in pigs in vivo. Circulation, 2004; 110:3055-3061.

7. Gotte G, Amelio E, Russo S, et al. Short time nonenzymatic nitric oxide synthesis from L-arginine and hydrogen peroxide induced by shock waves treatment. FEBS Lett, 2002; 520:153-155.

8. Frey A, Sønksen J \& Fode M. Low-intensity extracorporeal shockwave therapy in the treatment of postprostatectomy erectile dysfunction: a pilot study. Scandinavian Journal of Urology, 2016; 50:2, 123-127.

9. Yee $\mathrm{CH}$, Chan ES, Hou SS, et al. Extracorporeal shockwave therapy in the treatment of erectile dysfunction: a prospective, randomized, doubleblinded, placebo controlled study. Int J Urol, 2014; 21:1041-1045.

10. Gruenwald I, Appel B, and Vardi Y. Low-intensity extracorporeal shock wave therapy-A novel effective treatment for erectile dysfunction in severe ED patients who respond poorly to PDE5 inhibitor therapy. J Sex Med, 2012; 9:259-264.

11. Yee CH, Chan ES, Hou SS, et al. Extracorporeal shockwave therapy in the treatment of erectile dysfunction: a prospective, randomized, doubleblinded, placebo controlled study. Int J Urol, 2014; 21:1041-1045.

12. Olsen $A B$, Persiani $M$, Boie $S$, et al. Can lowintensity extracorporeal shockwave therapy improve erectile dysfunction? A prospective, randomized, double-blind, placebo-controlled study. Scand J Urol, 2015; 49:329-333.

13. Srini VS, Reddy RK, Shultz T, et al. Low intensity extracorporeal shockwave therapy for erectile dysfunction: a study in an Indian population. Can J Urol, 2015; 22:7614-7622.

14. Vardi Y, Appel B, Kilchevsky A, et al. Does low intensity extracorporeal shock wave therapy have a physiological effect on erectile function? Shortterm results of a randomized, doubleblind, sham controlled study. J Urol, 2012; 187:1769-1775. 